This is an author produced version of a paper published in Contact Dermatitis. This paper has been peer-reviewed but does not include the final publisher proof-corrections or journal pagination.

Citation for the published paper:

Ryberg, $\mathrm{K}$ and Isaksson, $\mathrm{M}$ and Gruvberger, $\mathrm{B}$ and Hindsén, $\mathrm{M}$ and Zimerson, E and Bruze, $\mathrm{M}$.

"Contact allergy to textile dyes in southern Sweden."

Contact Dermatitis, 2006 Jun; 54(6):313-21 doi:10.1111/j.0105-1873.2006.00733.x

Access to the published version may require journal subscription.

Published with permission from: Blackwell Synergy 


\section{CONTACT ALLERGY TO TEXTILE DYES IN SOUTHERN SWEDEN}

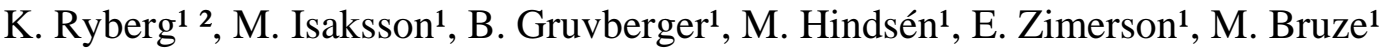

${ }^{1}$ Department of Occupational and Environmental Dermatology, Lund University, Malmö University Hospital, Malmö, Sweden. ${ }^{2}$ Department of Dermatology, NÄL, Trollhättan, Sweden.

Corresponding author

Kristina Ryberg

Address:

Department of Occupational and Environmental Dermatology, Malmö UniversityHospital, S20502 Malmö, Sweden.

Department of Dermatology, NÄL, S-461 85 Trollhättan, Sweden.

E-mail: Kristina.Morgardt-Ryberg@med.lu.se 


\begin{abstract}
Contact allergy to disperse dyes in textiles is documented in prevalence studies from southern Europe. To evaluate the prevalence of allergic patch test reactions to different textile dyes in southern Sweden, and to look at the sites of dermatitis in individuals hypersensitive to textile dyes, 3325 consecutively patch tested patients were retrospectively investigated. They had all been patch tested with the standard test series supplemented with a textile dye mix (TDM) consisting of 8 disperse dyes, i.e. Disperse (D) Blue 35, 106, and 124, D Yellow 3, D Orange 1 and 3, D Red 1 and 17. All but 3 of the TDM-positive patients were additionally tested with the separate dyes included in the mix. The frequency of contact allergy to TDM was $1.5 \%$, which is comparable with studies from southern Europe. The most common dye allergen was D Orange 1 . The high prevalence of allergic reactions to D Orange 1 was unexpected, whereas test reactions to D Blue 106 and 124 were lower than expected from other studies. Compared to all tested patients, the TDM-positive patients more often had dermatitis on their arms, face, neck and axillary folds, and women also had a higher frequency of hand dermatitis.
\end{abstract}

Key words: Contact allergy, patch testing, textile dye mix, disperse dyes, Disperse Orange 1, Disperse Blue 106, Disperse Blue 124, 1,4-phenylenediamine, black rubber mix, simultaneous reactivity. 


\section{Introduction}

Allergic reactions to textile dyes are documented, especially in prevalence studies from southern Europe (1-5). Such studies have not been performed in the Scandinavian countries. The most common dye sensitizers belong to the disperse dyes. They are used for colouring synthetic textile materials based on fibres such as polyester, acrylic, acetate and polyamide. The disperse dyes are planar and fat-soluble molecules with a substituted antraquinone or azo structure (Fig. 1) (6).

Contact allergy to textile dyes may result in a characteristic dermatitis but also in atypical signs, e.g. urticaria- and erythema multiforme-like lesions, and the causing allergen can easily be missed if the patients are not routinely tested with textile dyes (7).

Since 1999 all patients patch tested with the standard test series at the Department of Occupational and Environmental Dermatology and the Department of Dermatology, Malmö University Hospital, Malmö, Sweden were also tested with a textile dye mix (TDM) consisting of 8 disperse dyes, i.e. Disperse Blue (DB) 106, DB 35, DB 124, Disperse Yellow (DY) 3, Disperse Orange (DO) 1, DO 3, Disperse Red (DR) 1, and DR 17. Most of the TDM-positive patients were additionally tested with the separate dyes included in the mix. The aim of this retrospective study was to evaluate the prevalence of allergic patch test reactions to the TDM and to the separate dyes in the mix, to 1,4-phenylenediamine (PPD) and chemically related substances in southern Sweden from 1999 to 2003. We also wanted to study if there was a correlation between sensitization to textile dyes and clinical signs.

\section{Materials and Methods}

\section{Study population}

The study population consisted of 3325 patients consecutively tested at the departments from January 1999 to December 2003, 58.4\% women (mean age 47.2 years, range 10-90) and 41.6\% men (mean age 45.5 years, range $13-86$ ). 
Finn Chambers ${ }^{\circledR}$ (Epitest Ltd, Tuusula, Finland) on Scanpor ${ }^{\circledR}$ tape (Norgesplaster A/S, Vennesla, Norway) were used for the patch tests. The test chambers were left on the back for $48 \mathrm{~h}$ and readings followed ICDRG guidelines (8). Readings were performed on day (D) 3 or 4 and $\mathrm{D} 7$ or 8 .

The patients were all patch tested with the same standard series of the departments, including a mix of disperse dyes. The standard patch test series included PPD (1\% w/w pet.) and black rubber mix (BRM) (0.6\% w/w pet.), the latter consisting of 3 components, $\mathrm{N}, \mathrm{N}^{\prime}$-diphenyl-1,4phenylenediamine (DPPD), N-cyclohexyl-N'-phenyl-1,4-phenylenediamine (CPPD), and Nisopropyl-N'-phenyl-1,4-phenylenediamine (IPPD). Some of the patients were also tested with other patch test series and patient-supplied products depending on their history and skin signs. The composition of the dye mix was identical to a mix earlier used by Dr. Francisco Brandão, Almada, Portugal (personal communication) and consisted of DB 35, DY 3, DO 1, DO 3, DR 1, and DR17 (all 0.5\% w/w pet.), in addition to DB 106 and DB 124 (both 0.1\% w/w pet.), giving a total concentration of $3.2 \% \mathrm{w} / \mathrm{w}$ in pet. The patients who tested positively to the mix were tested with the 8 components separately. The dyes were bought from Chemotechnique Diagnostics, Malmö, Sweden, and the test preparations were made at the Department of Occupational and Environmental Dermatology. The mix and test preparations of the ingredients were made from the same batch. Some patients were also tested with a special textile colours and finish series from Chemotechnique Diagnostics. This series contains the same dyes as in the mix, but in higher concentrations, i.e. $1.0 \% \mathrm{w} / \mathrm{w}$ pet. each, and they are possibly made from different batches. 
Recording of data

The sites of dermatitis were documented before the patients were tested. For evaluation of the patch-test-positive population compared to all tested patients, we used Daluk, a data based registration system, where age, gender, contact allergies and site of dermatitis are recorded (9). The records of the patients with a positive reaction to TDM were reviewed.

\section{Statistical calculations}

To investigate possible overrepresentation of contact allergy to PPD and/or BRM in TDMpositive patients, Fisher's exact two-sided test was used. The same test was used to analyse whether the sites of dermatitis in TDM-positive patients were significantly different from the localisations of dermatitis in TDM-negative patients.

\section{Results}

\section{Patch test reactions}

50 patients (1.5\%), 28 women (mean age 49.6 years, range 24-72) and 22 men (mean age 48.9 years, range 15-66), reacted positively to the TDM. 65 of the tested patients (2.0\%; 46 women, 19 men) reacted to PPD and 12 of those (6 women, 6 men) were also allergic to TDM. 20 patients (0.6\%; 6 women, 14 men) reacted to BRM and 16 of those (4 women, 12 men) also reacted to the dye mix. 7 patients ( 3 women, 4 men) had simultaneous allergic reactions to TDM, PPD, and BRM, and all these patients had a +++ reaction to TDM. None of the tested patients reacted to both PPD and BRM without reacting to TDM. The pattern of concomitant reactivity to TDM, PPD, and BRM is shown in Fig. 2. In 4 patients reactions to the TDM were first seen on D 7. None of these patients reacted to PPD or BRM. The correlation between contact allergy to PPD and BRM, separately and combined, and +++ reaction to TDM was highly significant $(\mathrm{p}<0.001)$. Contact allergy to BRM and ++ reaction to TDM was also correlated $(\mathrm{p}<0.001)$. The test reactions to PPD, BRM, separately and combined, in relation to the test reactivity to TDM are shown in Fig. 3. 
The results of the ingredient testing are shown in Table $1.47 / 50$ patients were tested with the components of the TDM. 8 of them were tested both with the components in the mix and with the textile colours and finish series from Chemotechnique Diagnostics, which included the same dyes, but in a higher concentration, whereas 3 patients were tested only with the latter. The most frequent textile dye allergen when testing with the components of TDM was DO 1 (17/47 reacted positively; 36\%), followed by DY 3 (8 positive; 17\%), DB 124 and DR 1 (6 positive each; 13\%), DB 106 and DR 17 (5 positive each; 11\%), DB 35 (3 positive; 6\%), and DO 3 (1 positive; 2\%) (Fig. 4).

The pattern of concomitant reactivity to PPD, BRM, and to the components of the TDM is shown in Table 2. In the 17 patients allergic to DO 1, 14 reacted to BRM and 8 reacted to PPD, respectively. 4 of 8 patients allergic to DY 3 also reacted to PPD and 3 reacted to BRM. In the 6 patients reacting positively to DR 1,4 reacted to PPD and 2 reacted to BRM, respectively. 2 of the patients who reacted positively to DB 106 were also allergic to DB 124 . None of the patients who were allergic to DB 106 and/or 124 reacted to BRM or PPD (Table 2).

17 of the 47 TDM-positive patients had no positive reactions to the separate dyes of the mix tested in the lower concentration, but 8 had at least 1 doubtful (?) reaction. The TDM test reactivity in these 17 patients was + or ++.5 of the 17 patients were tested with the separate dyes in a higher concentration in the textile colours and finish series. 3 patients reacted to some ingredient (1 reacted to DB 106 and DB 124, 1 reacted to DO 1, and 1 reacted to DR 17).

A total of 11 patients were tested with the textile colours and finish series. 3 of the TDMpositive patients were tested only with this series. DB 106, DB 124, DO 1, and DY 3 were the most frequent dye allergens when tested in $1 \%$. 3 patients reacted positively to each of the aforementioned dyes, followed by DB 35, DR 1, and DR 17 (2 patients positive), and DO 3 (1 positive). 1 patient reacted positively to PPD and 2 reacted to BRM. These 3 patients also reacted to DO 1. 
The reactivity to the different components of the dye mix varied. Most of the test reactions to DB 106, DB 124, and DR 17 were weak, whereas most of the reactions to DO 1 were strong. Test reactivity to the different compounds of the dye mix is shown in Fig. 5.

\section{Correlation between contact allergy and localisation of dermatitis}

The sites of dermatitis in TDM-positive patients compared to skin site involvement in all tested patients are shown in Figure 6.

The most frequently involved skin sites in TDM-positive women were the hands (71\%), followed by face (54\%), arms (36\%), neck (14\%), trunk and axillae (both 11\%), scalp and feet (both 7\%), and legs (4\%). For TDM-positive men the most frequently involved skin sites were hands and arms (46\% each), followed by face (32\%), neck, trunk, legs, feet and genital area (all 9\%), scalp and axillae (both 5\%). For 3 of the TDM-positive patients there were no registrations of sites of dermatitis. Compared with all tested patients, TDM-positive patients more often had dermatitis on their arms, face, neck and axillary folds, and women also had a higher frequency of hand dermatitis. Overrepresentation of axillary dermatitis in women and underrepresentation of hand dermatitis in men were the only statistically significant associations noted ( $\mathrm{p}=0.03$ and $\mathrm{p}=0.02$, respectively). We have not been able to consider the influence of other factors of possible significance for localisation of dermatitis, e.g. atopic constitution, occupational exposure, and presence of additional contact allergies.

\section{Discussion}

In our study $1.5 \%$ of consecutively tested patients reacted positively to TDM, which is comparable to other studies from southern Europe (2-5). Among the TDM-positive patients concurrent positive reactions were seen to PPD and BRM. A positive correlation was noted between the intensity of TDM reactions and the proportion of positive reactions to PPD, BRM and simultaneous reactions to both substances (Fig. 3). The interpretation could be crossreactivity between some component(s) in the TDM, PPD and BRM, a common sensitizing 
metabolite, or an impurity present in all 3 patch test preparations. Simultaneous reactions to disperse dyes, especially DO 3, and PPD has previously been reported, and suspected to be either cross-sensitization or due to a common metabolite $(3,10)$. Concerning purity of textile dyes some have been analyzed with thin layer chromatography (11). The batches of investigated DY 3, DO 1, and DO 3 in the aforementioned study seemed to be pure, at least in the solvent system used, whereas DB 35, DR 1, and DR 17 were mixtures of dyes (11). This emphasises the need to analyze the purity of the textile dyes used for testing.

The frequency of skin lesions in the patients allergic to TDM in our study was high compared to 7 studies published in a review from Italy (12). In one of these studies (4), where Seidenari describes 100 subjects with textile dye dermatitis, the most frequent sites of dermatitis were the limbs, hands, and face followed by trunk, neck, axillae, and widespread dermatitis. In our study hands, face, and arms were more frequently involved than in the first mentioned study. Neck and axillary folds were involved just as often, and trunk and legs were affected more seldomly. According to the mentioned study (4) face, neck, and skin folds were significantly more frequently affected in women than in men, whereas widespread dermatitis was more common in men. The same pattern could be seen in our study, but according to our data widespread dermatitis was uncommon in both sexes. Statistically based comparisons of localisation of dermatitis in those with and without TDM-positivity may give clues on sensitization as well as clinical relevance of a demonstrated contact allergy. As our study was retrospective, information about clinical relevance was not always present. However, at present we are using a questionnaire which will give us such information in future studies. In our material, the only statistically significant correlates were seen for TDM-positive men with underrepresentation of hand dermatitis, and TDM-positive women with overrepresentation of axillary dermatitis. Although a higher value for the localisation of axillary dermatitis in TDM-sensitive women, as compared to men, this difference was not statistically significant. The underrepresentation of hand dermatitis in TDM-positive men is currently not understood, 
especially as there is an overrepresentation of hand dermatitis in women being TDM-positive, although not statistically significant $(\mathrm{p}=0.11)$. The overrepresentation of axillary dermatitis in TDM-positive women is supported by another Seidenari study (13), where involvement of skin folds were seen in $27 \%$ of disperse dye-positive women, mainly women testing positively to DB 106 and DB 124. This distribution of the dermatitis in TDM-positive women could be due to wearing tight-fitting underwear in synthetic textile materials leading to increased friction and sweating.

DO 1 was the most common dye allergen in our study, with a contact allergy rate of $0.5 \%$. When comparing the intensity of the patch test reactions to DO 1 with the reactions to the other disperse dyes in the mix, DO 1 shows a different pattern (Fig. 5). More individuals reacted with a +++ reaction compared to the reactivity to the other dyes. The possible significance, if any, of the various patterns of dose-response-relationship is presently not understood. Knowledge of the degree of exposure and the individual degree of reactivity is also necessary for the determination of possible clinical relevance.

We have not been able to find any reports on patch testing with DO 1 in consecutive dermatitis patients. The dye is mentioned in one study from Belgium, where aimed testing of 159 patients with suspected textile dye allergic dermatitis revealed $1.3 \%$ of the patients to be positive to DO 1 (14). In a retrospective study from Israel 1 of 55 patients with suspected textile dermatitis was test-positive to DO 1 (7). As mentioned before in one study, DO 1 seemed to be relatively pure (11). Our chemical analyses of DO 1 with thin layer chromatography and high performance liquid chromatography have given the same results. However, more studies need to be performed to find out the clinical relevance of allergic reactions to DO 1 and why DO 1 is rarely used for patch testing, although it seems to be a significant contact sensitizer.

In our study only 1 patient reacted to DO3, contrary to a study from Italy, where DO 3 and DB 124 gave the highest number of positive test reactions in adult patients (3). 1.7\% of 
consecutively tested Italian dermatitis patients reacted to DO3 and 66\% of the patients allergic to DO 3 were also positive to PPD. Our patient positive to DO 3 also reacted to PPD in addition to BRM.

$1.7 \%$ of the patients in the study from Italy were allergic to DB $124,16 \%$ of which were also allergic to PPD (3). In a later study by Seidenari (13) simultaneous contact allergy to DB106 and/or DB 124 and PPD was equally unusual. None of the patients in our study who were allergic to DB 106 and/or 124 were positive to PPD. Compared to earlier studies we found a lower prevalence of allergic reactions to DB106 and 124 than expected. When we tested these 2 substances in a higher concentration (1.0\% pet.), the number of patients positive to DB 106 and DB 124 increased from 11\% and 13\%, respectively, to 27\%. Most of the test reactions were weak testing with $0.1 \%$ pet. (Fig.5), compared to the reactions when testing with $1.0 \%$ pet. This emphasises the importance to define the optimal test concentration to detect contact allergy.

According to a study from Germany and Austria (15) an increase in the proportion of testpositive patients to DB 106 and DB 124 can be observed. Disperse blue dyes were even elected as “contact allergen(s) of the year” for 2000 in North America (16). Historically, PPD has been considered to be a screening allergen for textile dye dermatitis. With regard to our results a positive PPD test indicates simultaneous contact allergy in $0-70 \%$ depending on the dye. More recently, Canadian authors recommends DB 106 and 124 as screening substances in patients suspected of having textile dye dermatitis (17), which is in accordance with the findings from Germany and Austria (15). However, in our material DB 106 and DB 124 only traced $19 \%$ of the patients allergic to textile dyes, while PPD traced $26 \%$. The explanation is probably too low a concentration used testing DB 106 and DB 124 in our study.

17 of the TDM-positive patients did not test positively to any of the 8 ingredients when tested with the concentration used in the mix, as has been the case when testing other mixes e.g. 
fragrance-mix $(18,19)$. Possible explanations could be that the penetration into the skin of the TDM is higher, compared to the penetration of the ingredients when tested separately. Other possible explanations are a compound allergy, synergetic or additive effect of the different substances. Sometimes the reading of the TDM as well as the ingredients, especially DR, can be difficult, with a risk for overdiagnosing. Moreover, maybe a higher yield of positive patch test reactions to the 8 components of the TDM could be expected, if the ingredients had been tested at $1 \%$ pet. like in the textile colours and finish series from Chemotechnique instead of at lower concentration. However, testing with higher concentration of the respective substance is not a guarantee for a higher yield of positive reactions due to possible batch variations and possibly also different impurities.

The European Society of Contact Dermatitis recommends a sensitizer to be considered for inclusion in the standard series when routine testing of patients with suspected contact dermatitis results in a contact allergy rate exceeding $0.5-1 \%$ (20). The contact allergy rate found in our study raises the question whether a TDM should be included in the standard series. Before a decision further studies are needed to determine the purity of textile dyes, the implication of cross-sensitivity, and the relation between positive patch tests and clinical symptoms. The question remains whether women are primarily sensitized to PPD through hair dyes etc, and secondly react to TDM and whether men are primarily sensitized to BRM and secondly react to TDM. We also have to identify the textile dyes that are currently used, and to define their capacity to give allergic reactions.

\section{References}

1. Hatch K L, Maibach H I. Textile dye allergic contact dermatitis prevalence. Contact Dermatitis 2000: 42: 187-195.

2. Balato N, Lembo G, Avala F. Prevalence of textile dye contact sensitization. Contact Dermatitis 1990: 23: 111-126. 
3. Seidenari S, Mantovani L, Manzini B M, Pignatti M. Cross-sensitizations between azo dyes and para-amino compounds. Contact Dermatitis 1997: 36: 91-96.

4. Seidenari S, Manzini BM, Danese P: Contact sensitization to textile dyes: Description of 100 subjects. Contact Dermatitis 1991: 24: 253-258.

5. Lodi A, Ambonati M, Coassini A, Chiarelli G, Mancini L, Crosti C. Textile dye contact allergy in an allergic population. Contact Dermatitis 1989: 39: 314.

6. Le Coz C-J. Clothing. In: Textbook of Contact Dermatitis, $3^{\text {rd }}$ edition, Rycroft R J G, Menné T, Frosch P J, Lepoittevin J-P (eds): Springer-Verlag, Berlin. 2001: 725-749.

7. Lazarov A, Trattner A, David M, Ingber A. Textile Dermatitis in Israel: A Retrospective Study. Am J Cont Derm 2000: 11: 26-29.

8. Fregert S. Manual of contact dermatitis, $2^{\text {nd }}$ ed., Copenhagen. Munksgaard 1981, p. $71-76$.

9. Edman B. Computerized patch test data in contact allergy. Thesis. Malmö 1988.

10. Goon A T J, Gilmour N J, Basketter D A, White I R, Rycroft J G, Mcfadden J P. High frequency of simultaneous sensitivity to Disperse Orange 3 in patients with positive patch tests to p-phenylenediamine. Contact Dermatitis 2003: 48: 248.

11. Foussereau J, Dallara J M. Purity of standardized allergens: a thin layer chromatography study. Contact Dermatitis 1986: 14: 303-306.

12. Giusti F, Seidenari S. Disperse Dye Dermatitis: Clinical Aspects and Sensitizing Agents. Exogenous Dermatology 2003: 2: 6-10.

13. Seidenari S, Giusti F, Massone F, Mantovani L. Sensitization to disperse dyes in a patch test population over a five-year period. Am J Contact Dermat 2002: 13: 101-107.

14. Dooms-Goossens A. Textile dye dermatitis. Contact Dermatitis 1992: 27: 321-323.

15. Uter W, Geier J, Lessman H, Hausen B M. Contact allergy to Disperse Blue 106 and Disperse Blue 124 in German and Austrian patients, 1995 to 1999. Contact Dermatitis 2001: 44: 173-177. 
16. Storrs F J. Contact Allergen of the Year: Disperse Blue Dyes. Am J Cont Derm 2000: 11: $1-2$.

17. Pratt M, Taraska V. Disperse blue dyes 106 and 124 are common causes of textile dermatitis and should serve as screening allergens for this condition. Am J Cont Derm 2000: 11: 30-41.

18. Temesvari E, Nemeth I, Balo-Banga MJ, Husz S et al. Multicenter study of fragrance allergy in Hungary. Immediate and late type reactions. Contact Dermatitis 2002: 46: 325-330.

19. Frosch P J, Rastogi S C, Pirker C et al. Patch testing with a new fragrance mix reactivity to the individual constituents and chemical detection in relevant cosmetic products. Contact Dermatitis 2005: 52: 216-225.

20. Bruze M, Conde-Salazar L, Goossens A, Kanerva L, White I R. Thoughts on senzitizers in a standard patch test series. Contact Dermatitis 1999: 41: 241-250. 
Legends.

Fig. 1. Chemical structures of the 8 components in the textile dye mix, the 3 components in black rubber mix and p-phenylenediamine. (DB 35: Disperse Blue 35; DB 106: Disperse Blue 106; DB 124: Disperse Blue 124; DO 1: Disperse Orange 1; DO 3: Disperse Orange 3; DY 3: Disperse Yellow 3; DR 1: Disperse Red 1; DR 17: Disperse Red 17; DPPD: N,N'-diphenyl1,4-phenylenediamine; CPPD: N-cyclohexyl-N'-phenyl-1,4-phenylenediamine; IPPD: Nisopropyl-N'-phenyl-1,4-phenylenediamine; PPD: 1,4-phenylenediamine.)

Fig. 2. Number of patients with a positive patch test to textile dye mix (TDM), 1,4phenylenediamine (PPD), and black rubber mix (BRM), individually and combined, in the 3325 tested patients.

Fig. 3. Test reactions to 1,4-phenylenediamine (PPD) and black rubber mix (BRM), separately and combined, in the 3325 tested patients divided according to test reactivity to textile dye mix (TDM). The reactivity is described as negative (3165 patients), doubtful (?) (110 patients), + (14 patients), ++ (23 patients), ${ }^{+++}$(13 patients). ${ }^{* * *} \mathrm{p}<0.001,{ }^{*} \mathrm{p}<0.05$, n.s. $=$ not significant.

Fig.4. Numbers of patients with positive patch tests to the 8 disperse (D) dyes in the textile dye mix (TDM), to 1,4-phenylenediamine (PPD), and black rubber mix (BRM) in 47 patients testing positively to TDM.

Fig. 5. Test reactivity to the 8 different compounds of the dye mix in 47 patients testing positively to textile dye mix. The reactivity is described as doubtful (?),,,++++++ .

Fig. 6. Sites of dermatitis documented in Daluk. Comparison between patients positive to textile dye mix (TDM), and all tested patients, as well as gender differences.

Table 1. Patch test results in the 50 patients with a positive patch test to textile dye mix (TDM). Test reactivity to TDM, to the different disperse (D) dyes, 1,4-phenylenediamine (PPD), and black rubber mix (BRM) is described as,,++++++ . Not tested is described as *.

Table 2. Pattern of concomitant reactivity to the 8 disperse dyes in the textile dye mix, to $\mathrm{PPD}$, and BRM in the 47 patients testing positively to the mix.

(DB: Disperse Blue; DY: Disperse Yellow; DO: Disperse Orange; DR: Disperse Red; PPD: 1,4-phenylenediamine; BRM: black rubber mix.) 
Unspecified chemical formula according to Chemical Abstracts

DB 35<smiles>CCN(CCO)c1ccc(N=Nc2ncc([N+](=O)[O-])s2)c(C)c1</smiles>

DB 106<smiles>CCN(CCOC(C)=O)c1ccc(N=Nc2ncc([N+](=O)[O-])s2)c(C)c1</smiles>

\section{DB 124}<smiles>CNc1ccc([N+](=O)[O-])cc1</smiles><smiles>N=Nc1ccc(I)cc1</smiles>

DO 1<smiles>[R19][R19]</smiles><smiles>Cc1cc(N(CCO)CCO)ccc1N=Nc1ccc([N+](=O)[O-])cc1</smiles>

DR 17

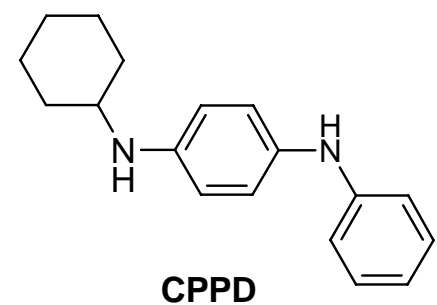<smiles>Nc1ccc(/N=N/c2ccc([N+](=O)[O-])cc2)cc1</smiles>

DO 3<smiles>CCN(CCO)c1ccc(/N=N/c2ccc([N+](=O)[O-])cc2)cc1</smiles>

DR 1
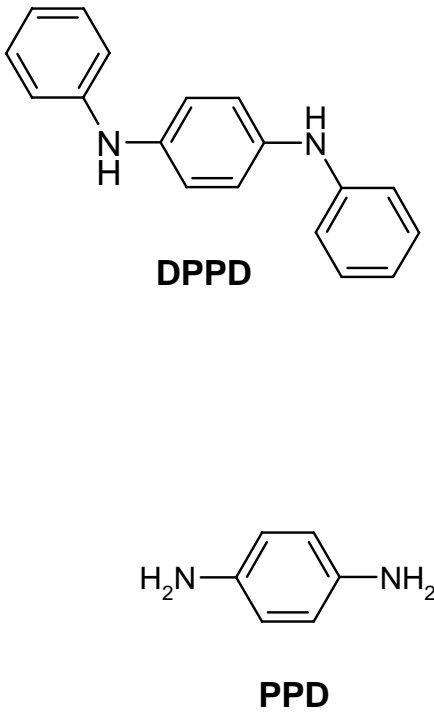


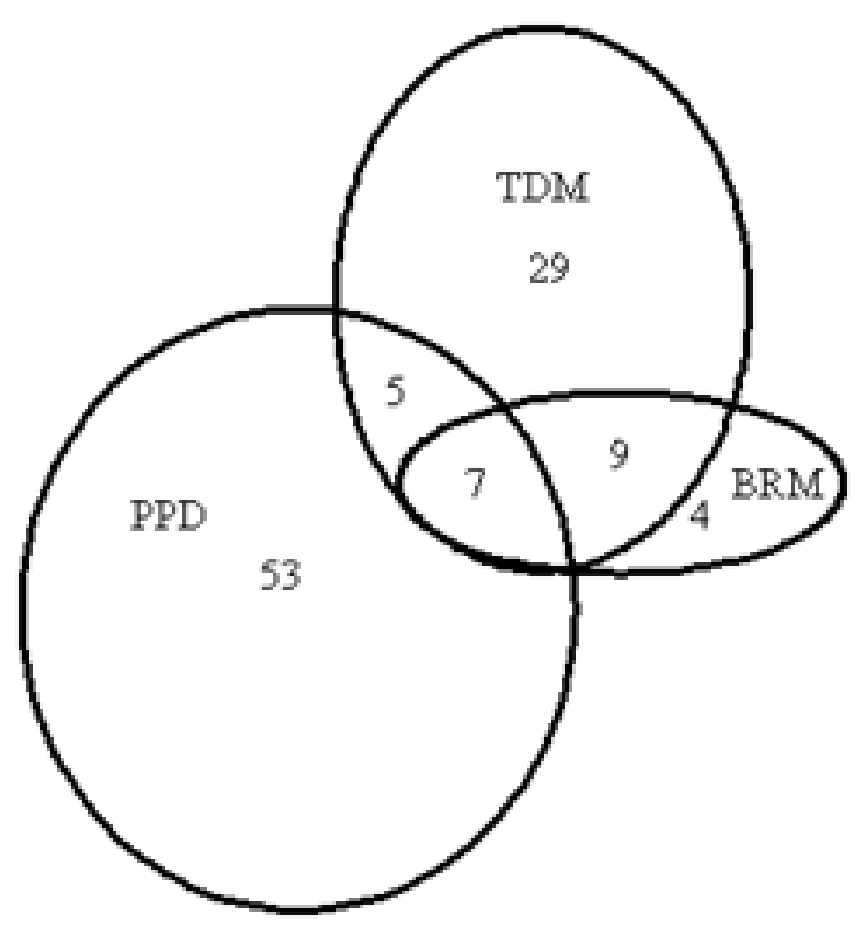



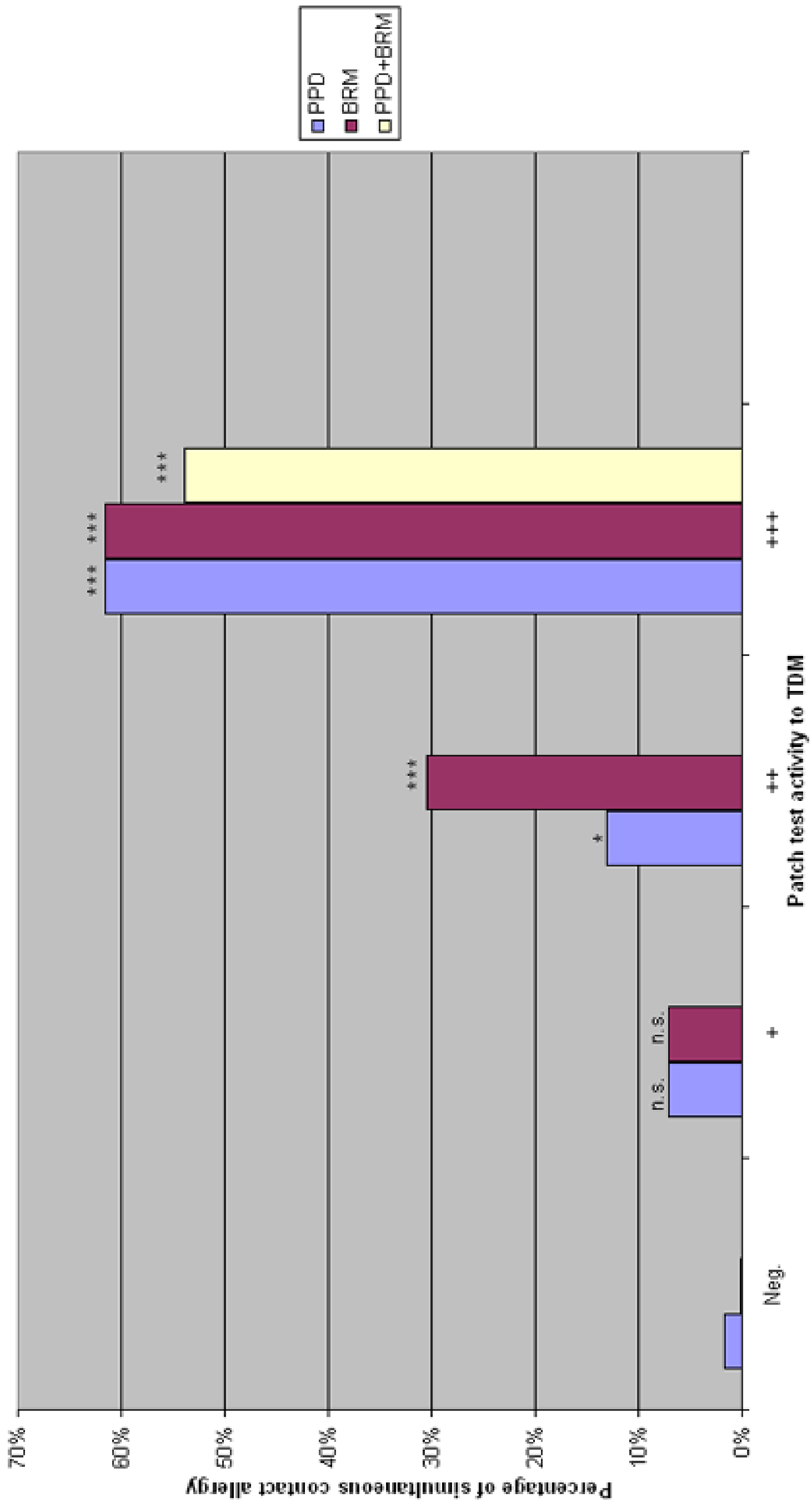


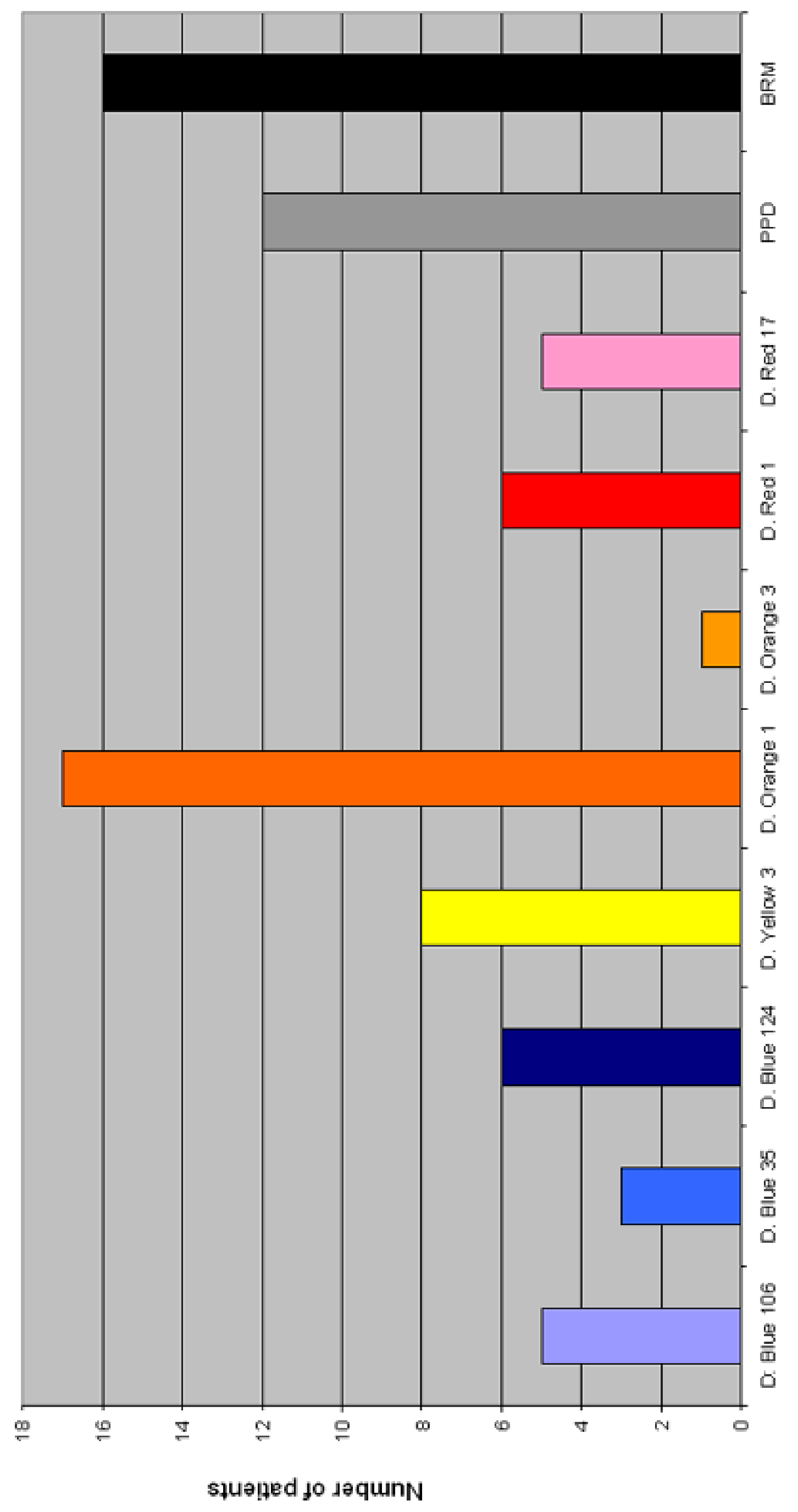




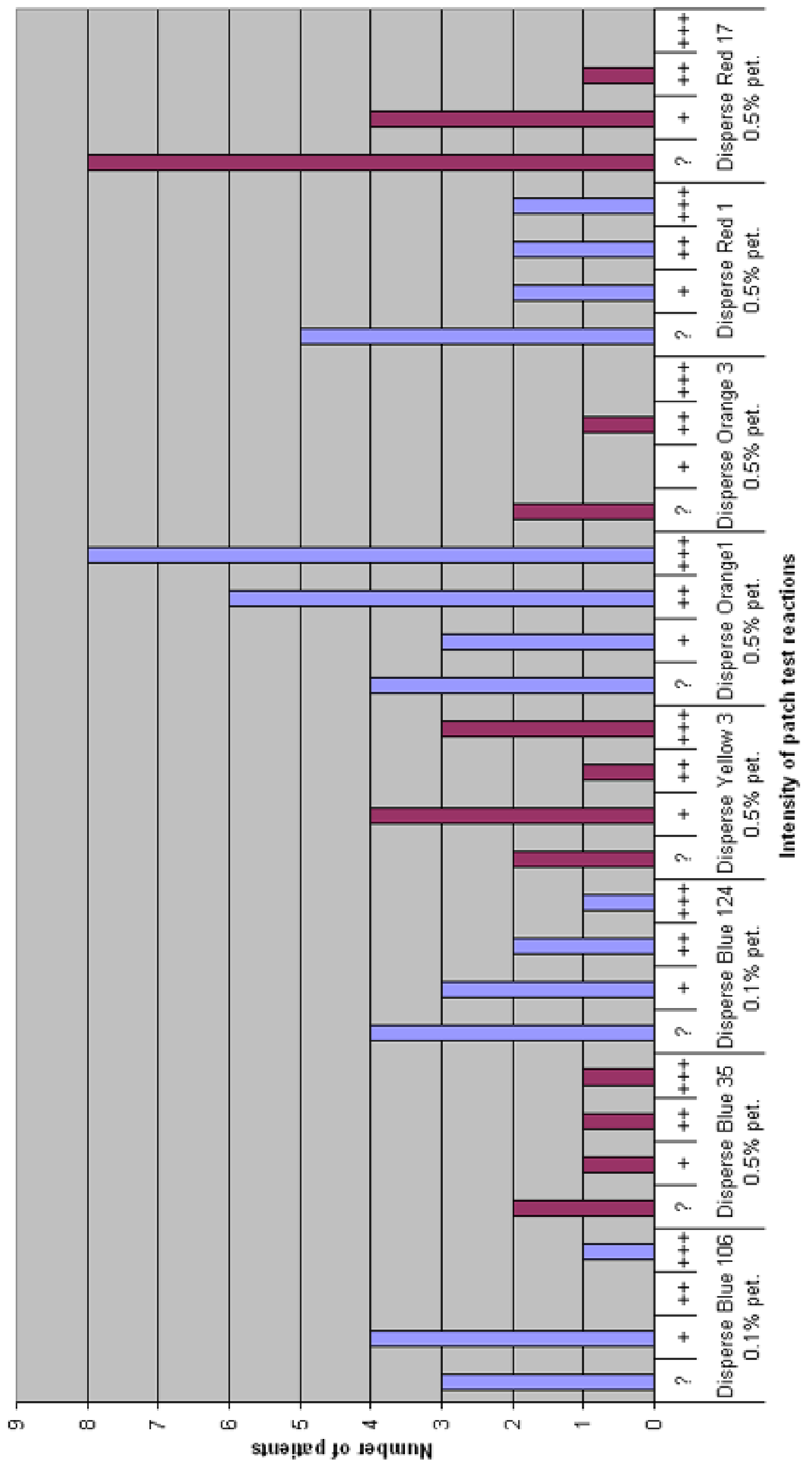



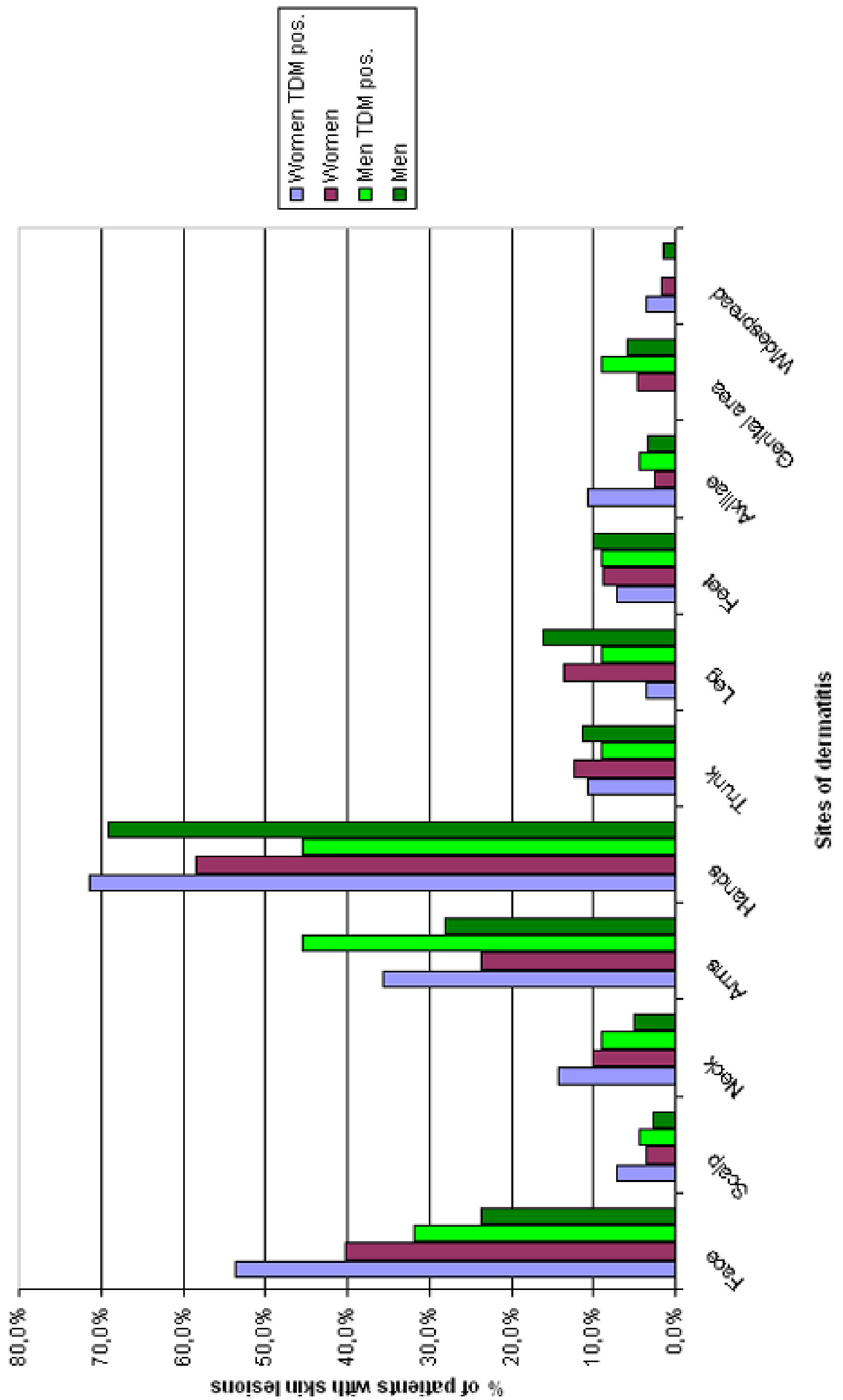


\begin{tabular}{|c|c|c|c|c|c|c|c|c|c|c|c|c|}
\hline $\begin{array}{l}x \\
0 \\
\omega\end{array}$ & $\begin{array}{l}a \\
\stackrel{0}{1}\end{array}$ & $\stackrel{\mathrm{s}}{\circ}$ & 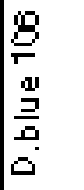 & 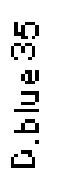 & 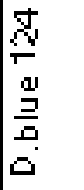 & 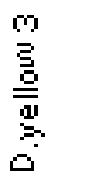 & 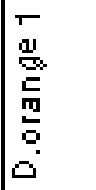 & 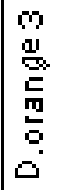 & 它 & 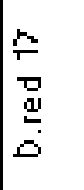 & $\frac{\circ}{0}$ & $\frac{\Sigma}{\text { 畄 }}$ \\
\hline$F$ & $40^{\circ}$ & +++ & & & & $x$ & & $x$ & 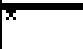 & $x$ & & \\
\hline$F$ & 43 & +++ & $\pi$ & $\bar{x}$ & $\pi$ & $\pi$ & $\bar{x}$ & $\pi$ & $\pi$ & $\pi$ & & \\
\hline$F$ & 48 & +++ & $\pi$ & $\bar{x}$ & $x$ & $\pi$ & $\pi$ & $\pi$ & $\pi$ & $\pi$ & & \\
\hline $\bar{F}$ & 40 & +++ & & +++ & & +++ & +++ & ++ & ++ & & +++ & ++ \\
\hline$M$ & 19 & +++ & & & & +++ & +++ & & +++ & ++ & +++ & ++ \\
\hline$F$ & 31 & +++ & & & & + & +++ & & +++ & + & +++ & \\
\hline$M$ & 45 & +++ & & & & & +++ & & & & + & +++ \\
\hline$M$ & 15 & +++ & & & & & +++ & & & & +++ & + \\
\hline$F$ & 47 & +++ & & & & & +++ & & & & ++ & +++ \\
\hline $\bar{F}$ & 39 & +++ & & & & & +++ & & & & +++ & +++ \\
\hline$|M|$ & 50 & +++ & & & & & ++ & & & & ++ & ++ \\
\hline$M$ & 59 & +++ & & & & & ++ & & & & & +++ \\
\hline$F$ & 30 & +++ & +++ & & +++ & & & & & & & \\
\hline $\mid \omega^{\prime}$ & 58 & ++ & & & & & +++ & & & & & ++ \\
\hline$F$ & 53 & ++ & & & & & ++ & & & & & \\
\hline$M$ & 51 & ++ & & & & & ++ & & & & & ++ \\
\hline $\mid \mathbf{M I}^{\prime}$ & 53 & ++ & & & & & ++ & & & & & ++ \\
\hline$M$ & 54 & ++ & & & & +++ & ++ & & & & & +++ \\
\hline$M$ & 59 & ++ & & ++ & & & + & & & & & + \\
\hline$F$ & 55 & ++ & & & & & + & & & & & +++ \\
\hline $\mathrm{F}$ & 54 & ++ & & & & ++ & & & ++ & & & \\
\hline $\bar{F}$ & 34 & ++ & & & & & & & & & & \\
\hline$F$ & 46 & ++ & & & & & & & & & +++ & \\
\hline $\bar{F}$ & 72 & ++ & + & + & & & & & & & & \\
\hline$F$ & 50 & ++ & & & & & & & & & & \\
\hline$F$ & 44 & ++ & & & ++ & & & & & & & \\
\hline$M$ & 49 & ++ & & & & & & & & & & ++ \\
\hline$M$ & 46 & ++ & & & ++ & & & & & & & \\
\hline $\mid \mathbf{M I}^{\prime}$ & 48 & ++ & & & & + & & & & & & \\
\hline$M$ & 40 & ++ & & & & & & & & & +++ & \\
\hline $\mathrm{F}$ & 43 & ++ & & & & + & & & + & + & +++ & \\
\hline$M$ & 54 & ++ & & & & & & & & & & \\
\hline$F$ & 49 & ++ & + & & & & & & & & & \\
\hline$M$ & 66 & ++ & & & & & & & & & & \\
\hline$F$ & 53 & ++ & & & & & & & & & & \\
\hline$M$ & 40 & ++ & & & & & & & & & & \\
\hline$F$ & 58 & + & & & & & + & & & & & \\
\hline $\bar{F}$ & 51 & + & & & + & + & & & & & & \\
\hline$F$ & 49 & + & + & & + & & & & + & + & & \\
\hline $\mathrm{F}$ & 60 & + & & & & & & & & & & \\
\hline $\bar{F}$ & 24 & + & & & & & & & & & & \\
\hline$M$ & 25 & + & & & & & & & & & & \\
\hline $\mathrm{F}$ & 29 & + & + & & & & & & & & & \\
\hline$M$ & 18 & + & & & & & & & & & +++ & \\
\hline$M$ & 55 & + & & & & & & & & & & ++ \\
\hline $\mathrm{F}$ & 46 & + & & & & & & & & + & & \\
\hline$M$ & 62 & + & & & + & & & & & & & \\
\hline $\mathrm{F}$ & 63 & + & & & & & & & & & & \\
\hline $\bar{F}$ & 51 & + & & & & & & & & & & \\
\hline$M$ & 38 & + & & & & & & & & & & \\
\hline
\end{tabular}




\begin{tabular}{|l|r|r|r|r|r|r|r|r|r|r|}
\hline & PPD & BRM & DE 106 & DE 35 & DE 124 & DY 3 & DO 1 & DO 3 & DR 1 & DR 17 \\
\hline PPD & $\mathbf{1 2}$ & 7 & 0 & 1 & 0 & 4 & 8 & 1 & 4 & 3 \\
\hline BRM & 7 & $\mathbf{1 6}$ & 0 & 2 & 0 & 3 & 14 & 1 & 2 & 1 \\
\hline DE 106 & 0 & 0 & $\mathbf{5}$ & 1 & 2 & 0 & 0 & 0 & 1 & 1 \\
\hline DE 35 & 1 & 2 & 1 & $\mathbf{3}$ & 0 & 2 & 2 & 1 & 1 & 0 \\
\hline DB124 & 0 & 0 & 2 & 0 & $\mathbf{6}$ & 1 & 0 & 0 & 1 & 1 \\
\hline DY 3 & 4 & 3 & 0 & 2 & 1 & $\mathbf{8}$ & 4 & 1 & 5 & 3 \\
\hline D01 & 8 & 14 & 0 & 2 & 0 & 4 & $\mathbf{1 7}$ & 1 & 3 & 2 \\
\hline DO 3 & 1 & 1 & 0 & 1 & 0 & 1 & 1 & $\mathbf{1}$ & 1 & 0 \\
\hline DR 1 & 4 & 2 & 1 & 1 & 1 & 5 & 3 & 1 & $\mathbf{6}$ & 4 \\
\hline DR 17 & 3 & 1 & 1 & 0 & 1 & 3 & 2 & 0 & 4 & $\mathbf{5}$ \\
\hline
\end{tabular}
.

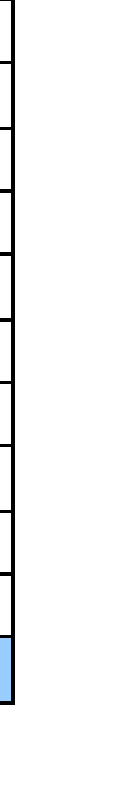
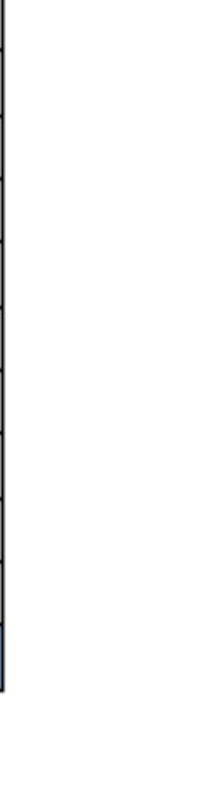
(⿸丆口 